\title{
ADRIEN DOUADY \\ Le théorème des images directes de Grauert [d'après Kiehl-Verdier]
}

Astérisque, tome 16(1974), p. 49-62

<http://www.numdam.org/item?id=AST_1974_16_49_0>

(C) Société mathématique de France, 1974, tous droits réservés.

L'accès aux archives de la collection « Astérisque » (http://smf4.emath.fr/ Publications/Asterisque/) implique l'accord avec les conditions générales d'utilisation (http://www.numdam.org/conditions). Toute utilisation commerciale ou impression systématique est constitutive d'une infraction pénale. Toute copie ou impression de ce fichier doit contenir la présente mention de copyright.

\section{Numdam}

Article numérisé dans le cadre du programme Numérisation de documents anciens mathématiques http://www.numdam.org/ 
LE THÉORÈME DES IMAGES DIRECTES DE GRAUERT

[d'après KIEHL-VERDIER]

par Adrien DOUADY

1. Introduction.

Soient $X$ un espace C-analytique séparé à base dénombrable et $\underline{F}$ un faisceau analytique coherent sur $X$. On sait que, pour tout ouvert $U$ de $X$, I'espace vectoriel $\underline{F}(U)$ est de façon naturelle un espace de Fréchet nucléaire. Si V est un ouvert relativenent compact de $U$, ce que nous écrirons $V$ CC $U$, la restriction $P: \underline{F}(U) \rightarrow \underline{F}(V)$ est nucléaire, donc compacte [6].

THÉORÈME de finitude [1].- Si X est compact, les espaces vectoriels $H^{n}(X ; \underline{F})$ sont de dimension finie sur $C$.

La démonstration de ce résultat utilise les ingrédients suivants : THÉOṘ̀ME de perturbation.- Soient $E$ et $F$ deux espaces de Fréchet, $f: E \rightarrow F$ un morphisme surjectif et $u: E \rightarrow F$ un morphisme compact. Alors $\operatorname{Coker}(f-u)$ est de dimension finie et séparé.

De ce théorème, on déduit facilement :

COROLLAIRE (L. Schwartz).- Soient $E^{\cdot}$ et $F^{\cdot}$ deux complexes d'espaces de Fréchet et $f^{*}: E^{*} \rightarrow F^{*}$ un morphisme induisant un isomorphisme sur I'homologie et tel que $f^{n}$ soit compact. Alors les espaces vectoriels $H^{n}\left(E^{*}\right)$ et $H^{n}\left(F^{\circ}\right)$ sont de dimension finie. 


\section{A. DOUADY}

$\mathrm{II}^{\mathrm{bi}}-\mathrm{O} \mathrm{O2}$

Pour démontrer le Thérème de finitude, on prend deux recouvrements finis $\underline{V}=\left(\mathrm{v}_{i}\right)$ et $\underline{W}=\left(\mathrm{w}_{i}\right)$ de $\mathrm{X}$ par des ouverts de Stein tels que $w_{i}$ cc $\mathrm{v}_{i}$ pour tout $i$. Posons $E^{*}=C^{*}(X, \underline{V} ; \underline{F})$ et $F^{*}=C^{*}(X, \underline{W} ; \underline{F})$. D'après le Theorème $B$ de Cartan et le Théorème de Leray, on a $H^{n}\left(E^{\circ}\right)=H^{n}\left(F^{\circ}\right)=H^{n}(X ; F)$. Comme la restriction $P: E^{*} \rightarrow F^{*}$ est compacte, le Théorème de finitude découle du résultat de Schwartz.

on notera qu'on utilise seulement la compacité de $\rho$, non sa nucléarité. Le Theorème des images directes est une "version relative" du théoreme de finitude :

THÉORÈME des images directes.- Soient $X$ et $S$ des espaces C-analytiques séparés, $\pi: X \rightarrow S$ un morphisme propre et $F$ un faisceau cohérent sur $X$. Alors les faisceaux $R^{n} \pi_{*} F$ sont cohérents.

Rappelons que $R^{n} \pi_{*} F$ est le faisceau sur $S$ associé au préfaisceau $\mathrm{U} \mapsto \mathrm{H}^{\mathrm{n}}\left(\pi^{-1}(\mathrm{U}) ; \mathrm{F}\right)$.

Une démonstration de cet énoncé a été donnée par Grauert en 1960 (cf. [2]). Une tentative a été faite pour l'exposer au Séminaire Bourbaki en Mai 1961. En fait, la démonstration de Grauert était extrênement pénible et comportait de nombreuses erreurs de détail. Il a fallu attendre 1969 pour que Knorr [3] donne une démonstration correcte, mais toujours pénible. Au Congrès de Nice, en 1970, Kiehl a exposé le principe d'une nouvelle démonstration. Cette démonstration a été réalisée par Kiehl-Verdier [4]. Forster-Knorr [5] ont également obtenu une démonstration élémentaire.

C'est la méthode de Xiehl-Verdier que nous rapportons ici. Elle suit le plan de la démonstration du Théorème de finitude, mais en considérant des modules de 


$$
\text { II }^{\text {bis }}-03
$$

Fréchet sur des algébres de Fréchet, et en exploitant la nucléarité. Il faut introduire une notion de nucléarité relativement à une algèbre ( 1 'algèbre $\underline{O}(s)$ ), notion qui decrive les proprietés des restrictions $\rho: \underline{F}(U) \rightarrow \underline{F}(V)$ pour $\mathrm{V} \subset \mathrm{U} \subset \mathrm{X}$ et $\mathrm{V}$ relativement s-propre dans $\mathrm{U}$. Ceci nécessite de continuels changements d'algèbre, qui correspondent a des rétrécissements de la base $s$.

A titre d'application, nous indiquons a la fin une démonstration du theoreme de semi-continuité.

\section{Les foncteurs $\hat{\theta}_{A}$ et $\hat{\operatorname{Tor}}_{n}^{A}$.}

Dans ce $n^{\circ}$ et le suivant, A designe une algebre de Frtchet nucleaire, i.e. un espace de Fréchet nucléaire sur C muni diune multiplication bilinéaire continue qui en fait une algebre commutative associative et unifere. On appelle A-module de Frechet nucleaire un espace de Fréchet nucleaire $\mathbf{E}$ sur $C$ muni d'une loi bilinéaire continue $A \times E \rightarrow E$ qui en fait un A-module. on dit qu'un A-module de Fréchet est nucleairement libre s'il est isomorphe à un A-module de la forme $A \hat{\otimes} V$, où $V$ est un espace de Fréchet nucléaire sur $C$. Soit $E$ un A-module de Fréchet nucléaire; on appelle résolution nucléairement libre de $E$ une suite exacte

$$
\ldots \rightarrow I_{n} \stackrel{d_{n}}{\rightarrow} I_{n-1} \rightarrow \ldots \rightarrow L_{0} \rightarrow E \rightarrow 0,
$$

de A-modules de Fréchet, où les $L_{i}$ sont nucléairement libres. Une telle résolution est dite directe $s i$, pour tout $n$, l'image de $d_{n}$ admet un supplémentaire topologique dans $L_{n-1}$ comme espace vectoriel sur C (pas comme A-module):

PROPOSITION 1.- a) Tout A-module de Fréchet nucléaire admet une résolution nucléai rement libre directe. 


\section{A. DOUADY}

$\mathrm{II}^{\mathrm{bis}}-04$

b) Soient L. et I: deux résolutions nucléairement libres directes d'un Amodule E. Il existe un morphisme de $L:$ dans L. unique à homotopie près.

Démonstration. a) Elle répond à la question, la "résolution standard" de E, qu'on obtient en posant $I_{n}=\underbrace{A \hat{\otimes} \ldots \hat{\otimes} A}_{n+1} \hat{\otimes} E$, la structure de A-module provenant du premier facteur, et

$d_{n}\left(a_{0} \otimes \ldots \otimes a_{n} \otimes x\right)=\Sigma(-1)^{i} a_{0} \otimes \ldots \otimes a_{i} a_{i+1} \otimes \ldots \otimes a_{n} \otimes x+(-1)^{n} a_{0} \otimes \ldots \otimes a_{n-1} \otimes a_{n} x ;$ un opérateur d'homotopie, C-linéaire seulement, est donné par $t \mapsto 1 \otimes t$.

b) Si $L=A \otimes V$, on a $L_{A}(L ; F)=C_{C}(V ; F)$ pour tout A-module de Fréchet $F$, donc $L$ est projectif au sens suivant $:$ si $F \rightarrow G \rightarrow 0$ est une suite exacte, directe $(\operatorname{sur} C)$, de A-modules de Fréchet, $\mathbf{C}_{A}(L ; F) \rightarrow L_{A}(L ; G)$ est surjectif. L'assertion $b$ ) en résulte de façon classique.

Soient $E$ et $F$ deux A-modules de Fréchet nucléaires. On pose $E \hat{\otimes}_{A} F=\operatorname{Coker}(d: E \hat{\otimes} A \hat{\otimes} F \rightarrow E \hat{\otimes} F)$, où $d(x \otimes a \otimes y)=a x \otimes y-x \otimes a y$. L'espace $E \hat{\otimes}_{A} F$ est un A-module non nécessairement séparé. Il est séparé si $E$ est nucléairement libre, car si $E=A \hat{\otimes}_{C} V$, on a $E \hat{\otimes}_{A} F=V \otimes_{C} F$.

Pour tout $n$, on définit $\operatorname{Tôr}_{n}^{A}(E, F)$ de la façon suivante : on prend une résolution $L$. $\rightarrow F$ nucléairement libre directe de $F$ et on pose $\operatorname{Tôr}_{n}^{A}(E, F)=H_{n}\left(E \hat{\otimes}_{A} L_{0}\right) ;$ c'est un A-module non nécessairement séparé qui, $a$ isomorphisme canonique près, ne dépend pas du choix de L. ; on a $\operatorname{Tor}_{0}^{A}(E, F)=E \hat{\otimes}_{A} F$.

on a $\operatorname{Tor}_{q}^{A}(E, F)=0$ pour $q>0$ si $F$ est nucleairement libre, car on peut alors prendre $L$. réduite à $L_{0}=F$. Si $0 \rightarrow E_{1} \rightarrow E_{2} \rightarrow E_{3} \rightarrow 0$ est une suite exacte courte de A-modules de Fréchet nucléaires, on a une suite exacte courte de complexes $0 \rightarrow E_{1} \hat{\otimes}_{A}$ L. $\rightarrow E_{2} \hat{\otimes}_{A}$ L. $\rightarrow E_{3} \hat{\otimes}_{A}$ L. $\rightarrow 0$, d'où une 


\section{IMAGES DIRECTES}

$$
\text { II }^{\text {bis }}-05
$$

suite exacte (algébrique) longue des $\operatorname{Tôr}_{n}$. En prenant pour L. la résolution standard de $F$, on obtient $E \hat{\otimes}_{A} L_{n}=E \hat{\otimes} \underbrace{A \otimes A}_{n} \otimes F$, et on constate la symétrie des bifoncteurs $\operatorname{Tôr}_{n}^{A}$. De tout cela on déduit que l'on peut calculer les $\operatorname{Tor}_{n}^{A}(E, F)$ en prenant une résolution rucléairement libre non nécessairement directe de $F$.

\section{Transversalité.}

DÉFINITION 1.- Soient $E$ et $F$ deux A-modules de Fréchet nucléaires. On dit que $E$ et $F$ sont transverses si $E \hat{\theta}_{A} F$ est séparé et si $\operatorname{Tôr}_{q}^{A}(E, F)=0$ pour $q>0$.

Si $E$ est nucléairement libre, il est transverse à $F$ pour tout $F$. Soit $0 \rightarrow E_{1} \rightarrow E_{2} \rightarrow E_{3} \rightarrow 0$ une suite exacte, si $F$ est transverse a $E_{2}$ et $E_{3}$, il est transverse à $E_{1}$.

Soient $\mathrm{A} \rightarrow \mathrm{A}_{1} \rightarrow \mathrm{A}_{2}$ des homomorphismes d'algèbres nucléaires et $\mathrm{E}$ un A-module de Fréchet nucléaire. Si $A_{1}$ et $A_{2}$ sont transverses a $E$ sur $A$, alors $A_{2}$ est transverse a $A_{1} \hat{\otimes}_{A} E$ sur $A_{1}$.

PROPOSITION 2.- Soient $S_{0}$ un espace de Stein, $U_{0}$ un ouvert de stein de $c^{n}$ et $F$ un faisceau analytique cohérent sur $S_{0} \times U_{0}$. Soient $S \subset C S_{0}$ et $U \subset \subset U_{0}$ des ouverts de Stein, et $S^{\prime}$ un ouvert de Stein de $S$. Alors $\underline{O}\left(S^{\prime}\right)$ et $\underline{F}(S \times U)$ sont transverses sur $\underline{O}(S)$.

Démonstration. L'espace de Fréchet $\underline{O}(S \times U)=\underline{O}(S) \hat{O} \underline{O}(U)$ est un module nucléairement libre sur $\underline{O}(s)$. Soit $\underline{L} . \rightarrow \underline{F}$ une résolution libre de $\underline{F}$ sur $S \times U$ (une telle résolution existe d'après le Théorène $A$ ). D'après le Théorème $B$, $\underline{L} \cdot(S \times U)$ est une résolution de $\underline{F}(S \times U)$, nucléairement libre sur $\underline{O}(S)$. on a 


\section{A. DOUADY}

II $^{\mathrm{bis}}-06$

$\underline{o}\left(S^{\prime}\right) \hat{\otimes}_{\underline{O}}(S) \underline{O}(S \times U)=\underline{o}\left(S^{\prime}\right) \hat{\otimes}_{C} \underline{o}(U)=\underline{O}\left(S^{\prime} \times U\right)$, d'où

$\underline{O}\left(S^{\prime}\right) \hat{\theta}_{O}(S) \underline{L} \cdot(S \times U)=\underline{L} \cdot\left(S^{\prime} \times U\right)$. Mais $\underline{L} \cdot\left(S^{\prime} \times U\right)$ est une résolution de

$\underline{F}\left(S^{\prime} \times U\right)$, d'où $\underline{O}\left(S^{\prime}\right) \hat{\otimes}_{0}(S) \underline{F}(S \times U)=\underline{F}\left(S^{\prime} \times U\right)$ et $\operatorname{Tôr} \frac{o}{q}(S)\left(\underline{o}\left(S^{\prime}\right), \underline{F}(S \times U)\right)=0$

pour $q>0$.

C.Q.F.D.

PROPOSITION 3.- Soient $E^{*}$ un complexe borné à droite de A-modules de Fréchet nucléaires, et $F$ un A-module de Fréchet nucléaire, transverse aux $E^{n}$. Si $E^{*}$ est acyclique en degré $2 k$, il en est de meme de $F \hat{\otimes}_{A} E^{*}$.

Démonstration. Les suites exactes $\rho \rightarrow z^{n}\left(E^{*}\right) \rightarrow E^{n} \rightarrow z^{n+1}\left(E^{*}\right) \rightarrow 0$

montrent par récurrence descendante que $Z^{n}\left(E^{\circ}\right)$ est transverse à $F$ pour $n \geq k-1$. Ces suites restent donc exactes par $\otimes_{A} F$.

COROLLAIRE.- Soient $E^{*}$ et $F^{*}$ deux complexes bornés à droite de A-modules de Fréchet nucleaires, $f: E^{*} \rightarrow F^{*}$ un morphisme et $M$ un A-module de Frechet nucleaire transverse aux $E^{n}$ et aux $F^{n}$. Si $f$ induit un isomorphisme sur l'homologie, il en est de meme de $1 \otimes f: M \otimes_{A} E^{*} \rightarrow M_{A} F^{*}$.

S'obtient en appliquant la proposition au "mapping cylinder" de $f$.

\section{Applications A-nucléaires et A-sous-nucléaires.}

DÉFINITION 2.- Soient $A$ une algèbre de Fréchet, $E$ et $F$ deux A-modules de Fréchet et $f: E \rightarrow F$ une application A-inéaire. On dit que $f$ est $A-$ nucléaire s'il existe une famille équicontinue $\left(\xi_{i}\right)$ d'applications A-linéaires de $E$ dans $A$, une famille bornée $\left(y_{i}\right)$ d'éléments de $F$ et une famille absolument sommable $\left(\lambda_{i}\right)$ de nombres complexes telles que, $\forall x \in E$,

$$
f(x)=\Sigma \lambda_{i} \xi_{i}(x) y_{i} \text {. }
$$




$$
\text { II }^{\text {bis }}-07
$$

On dit que $f$ est A-sous-nucléaire s'il existe un A-module de Fréchet $M$ et un diagramme commutatif

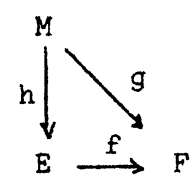

où $h$ est surjectif et $g$ est A-nucléaire.

Si $u: E \rightarrow F$ est A-nucléaire, et si $f: E_{1} \rightarrow E$ et $g: F \rightarrow F_{1}$ sont A-linéaires continues, $g \circ u \circ f$ est A-nucléaire. Si $v$ et $w$ sont des espaces de Fréchet nucléaires et $u: V \rightarrow W$ une application C-nucléaire, ${ }^{1} A \otimes: A \hat{\otimes} V \rightarrow A \hat{\otimes} W$ est A-nucléaire. Par exemple :

PROPOSITION 4.- Soient $S$ un espace de Stein, $U$ et $V$ deux ouverts de Stein de $C^{n}$ tels que $V \subset \subset U$. Alors la restriction $\rho: \underline{o}(S \times U) \rightarrow \underline{o}(S \times V)$ est $\underline{o}(S)$-nucléaire. $S_{i} S \subset \subset S_{0}$ et $U \subset \subset U_{0}$, où $S_{0}$ et $U_{0}$ sont de $S t e i n$, pour tout faisceau analytique coherent $E$ sur $S_{0} \times U_{0}$, la restriction $p: \underline{F}(S \times U) \rightarrow \underline{F}(S \times V)$ est $\underline{o}(S)$-sous-nucléaire.

Soient $E$ et $F$ deux A-modules de Fréchet et $f: E \rightarrow F$ une application A-linéaire. Soit $F_{1}$ un sous-A-module fermé de $F$ tel que $f(E) \subset F_{1}$ et notons $f_{1}$ I'application $x \mapsto f(x)$ de $E$ dans $F_{1}$. Si $f$ est A-nucléaire, $f_{1}$ n'est pas A-sous-nucléaire en général (meme si A, E et $F$ sont nucléaires). Cependant :

PROPOSITION 5.- Avec les notations ci-dessus, supposons $A, E$ et $F$ nucléaires. Soient $B$ une algèbre de Fréchet nucléaire et $P: A \rightarrow B$ un homomorphisme C-nucléaire d'algèbres. On suppose que $B$ est transverse sur $A$ à $E, F$ et $F / F_{1} \cdot$ Alors, si $f$ est A-sous-nucleaire, ${ }_{B} \otimes f_{1}: B \hat{\otimes}_{A} E \rightarrow B \hat{\otimes}_{A} F_{1}$ est B-sous-nucléaire. 


\section{A. DOUADY}

II $^{\text {bis }}-08$

Lemme 1.- $\underline{\text { Si }} f$ est A-nucléaire, $\rho \otimes f_{1}: E \rightarrow B \hat{\otimes}_{A} F_{1}$ est C-nucléaire.

Démonstration du lemme. Ecrivons $f(x)=\Sigma \lambda_{i} \xi_{i}(x) y_{i}$ comme dans la définition 2, et factorisons $p$ en $A \rightarrow A_{1} \stackrel{P_{1}}{\longrightarrow} B_{1} \rightarrow B$, où $A_{1}$ et $B_{1}$ sont des espaces de Banach et $\rho_{1}$ est nucléaire. Alors $\rho \otimes f: E \rightarrow B \hat{\otimes}_{A} F$ se factorise en $E \stackrel{u}{\longrightarrow} \ell^{\infty}\left(A_{1}\right) \stackrel{R}{\longrightarrow} \ell^{1}\left(B_{1}\right) \stackrel{v}{\longrightarrow} B \hat{\otimes}_{A} F$, où $u$ est dớnée par les $\xi_{i}$, $\dot{v}$ par les $y_{i}$, et $R=\Sigma \lambda_{i}{ }^{2}{ }_{i} \circ \rho_{1} \circ \pi_{i}$ est nucléaire. Donc $\rho \otimes f$ est C-nucléaire de $E$ dans $B \hat{\otimes}_{A} F$. Grace aux hypothèses de transversalite, $B \hat{\otimes}_{A} F_{1}$ s'identifie à un sous-espace fermé de $B \hat{\otimes}_{A} F$ et $\rho \otimes f$ applique $E$ dans ce sous-espace, l'application induite étant $p \otimes f_{1}$. Comme $E$ est nucléaire, il en résulte que $\rho \& f_{1}$ ist nucléaire.

Démonstration de la proposition. Soit

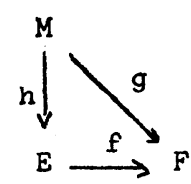

un diagramme commutatif où $h$ est surjectif et $g$ est A-nucléaire. Alors $g$ induit une application $g_{1}: M \rightarrow F_{1}$, et on a un diagramme commutatif

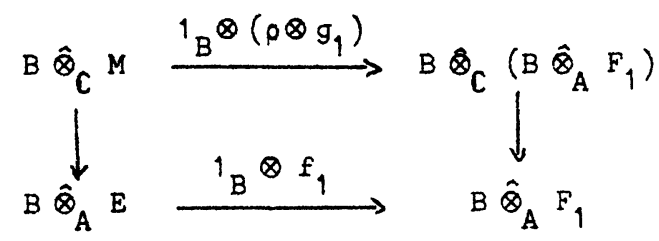

qui montre que $1_{B} \otimes f_{1}$ est A-sous-nucléaire. 


\section{Perturbations A-sous-nucléaires.}

THÉORÈME 1.- Soient A une algèbre de Fréchet nucléaire, $E$ et $F$ des Amodules de Fréchet nucléaires, f et $u$ deux morphismes de $E$ dans $F$. On suppose que u est A-suus-nucléaire, et que $f$ est surjectif. Soient $A_{1}$ une algèbre de Fréchet et $\rho: A \rightarrow A_{1}$ un homomorphisme d'algèbres qui se factorise à travers une algebre de Banach B. On suppose $A_{1}$ transverse sur $A$ a $E$ et $F$. Alors le conoyau de $1 \otimes(f-u): A_{1} \hat{\otimes}_{A} E \rightarrow A_{1} \hat{\otimes}_{A} F$ est un $A_{1}$-module de type fini.

(La conclusion de ce théorène est purement algébrique; le conoyau en question n'est pas nécessairement séparé.)

Lemme 2.- Soient B une algèbre de Banach, $M$ un B-module de Fréchet et $v: M \rightarrow M$ un morphisme B-nucléaire. Alors Coker $\left(1_{M}-v\right)$ est un Bmodule de type fini.

Démonstration. Ecrivons $v(x)=\Sigma \lambda_{i} \xi_{i}(x) y_{i}$ comme dans la definition 2 et factorisons $v$ en $M \stackrel{\alpha}{\longrightarrow} \ell^{1}(B) \stackrel{\beta}{\longrightarrow} M$, où $\alpha(x)=\left(\lambda_{i} \xi_{i}(x)\right)$ et $\beta$ est défini par les $y_{i}$. Posons $w=\alpha \circ \beta: \ell^{1}(B) \rightarrow \ell^{1}(B)$. L'application $w$ est $B-$ nucléaire car $\alpha$ l'est, et on a $\operatorname{Coker}\left(1_{M}-v\right) \approx \operatorname{Coker}\left(1_{\ell^{1}(B)}-w\right)$. On est donc ramené au cas où $M$ est un module de Banach.

$\mathrm{Si} M$ est un module de Banach, on peut mettre $v$ sous la forme $v^{\prime}+v^{\prime \prime}$, où $v^{\prime}$ est de rang fini sur $B$ et $\left\|v^{\prime \prime}\right\|<1$. Alors $1-v^{\prime \prime}$ est un automorphisme et $1-v=\left(1-v^{\prime \prime}\right)-v^{\prime}$ a un conoyau de type fini.

Démonstration du Théorème. a) on peut supposer que u est A-nucléaire et $E$ nucléairement libre. 


\section{A. DOUADY}

II $^{\text {bis }}-10$

b) On peut alors factoriser $u$ en $f \circ v$, où $v: E \rightarrow E$ est A-nucléaire. En effet, écrivons $u(x)=\Sigma \lambda_{i} \xi_{i}(x) y_{i}$ comme dans la définition 2, et $\lambda_{i}^{*}=\lambda_{i}^{\prime} \lambda_{i}^{\prime \prime}$ aver $\left(\lambda_{i}^{\prime}\right)$ sommable et $\left(\lambda_{i}^{\prime \prime}\right)$ tendant vers 0 . Quitte a remplacer $y_{i}$ par $\lambda_{i}^{\prime \prime} y_{i}$, on peut supposer que les $y_{i}$ tendent vers 0 . On peut alors écrire $y_{i}=f\left(x_{i}\right)$, où les $x_{i}$ tendent vers 0 , „t on $a u=f \circ v$, où $v(x)=\Sigma \lambda_{i} \xi_{i}(x) x_{i}$.

c) On peut supposer que $F=E$ et $f=1_{E}$. En effet, si $u=f \circ v, f$ donne une application surjective de $\operatorname{Coker}\left(1_{E}-v\right)$ sur Coker $(f-u)$, et de même après $\hat{\otimes}_{\mathrm{A}} \mathrm{A}_{1}$.

d) Supposons donc que $\rho: A \rightarrow A_{1}$ se factorise à travers une algèbre de Banach $B$, que $E=A \hat{\otimes}_{C} V$, où $V$ est un espace de Fréchet nucléaire et que $u: E \rightarrow E$ est A-nucléaire. Alors $B \hat{\otimes}_{A} E=B \hat{\otimes}_{C} V$ est un B-module de Fréchet,

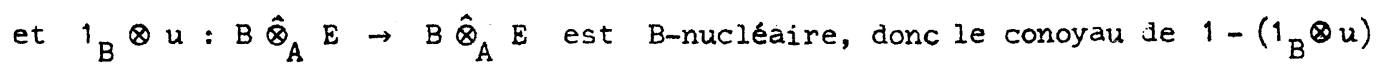
est un $B$-module de type fini. Autrement dit, il existe $r \in N$ et $h: B^{r} \rightarrow B \hat{\otimes}_{A} E$ tel que $\left(1_{B} \otimes(1-u), h\right): B \hat{\otimes}_{A} E \oplus B^{r} \rightarrow B \hat{\otimes}_{A} E$ soit surjectif. Alors $\left(1_{A_{1}} \otimes(1-u), 1_{A_{1}} \otimes h\right): A_{1} \hat{\otimes}_{A} E \oplus A_{1}^{r} \rightarrow A_{1} \hat{\otimes}_{A} E$ est surjectif. C.Q.F.D.

6. Un Théorème "a la Schwartz".

La démonstration du Théorème 2 comporte une récurrence utilisant le Théorème 1 et la Proposition 5, donc un grand nombre d'extensions des scalaires. Ceci nous amène à poser la définition suivante :

DÉFINITION 3.- On appelle chaîne nucléaire d'algèbres un système inductif $A=\left(\left(A_{t}\right),\left(\rho_{t}^{t^{\prime}}\right)\right)_{t \in[0,1] \text { où les }} A_{t}$ sont des algèbres de Fréchet nucléaires 
et où, pour $t^{\prime}<t^{\prime}, \rho_{t}^{t^{\prime}}: A_{t} \rightarrow A_{t^{\prime}}$ est un homomorphisme d'algèbres, C-nucléaire et se facturisant à travers une algèbre de Banach.

Si $E$ est un $A_{0}$-module de Fréchet nucléaire, on dit que $A_{-}$est transverse a $E$ si $A_{t}$ est transverse à $E$ sur $A_{0}$ pour tout $t$.

Exemple.- Soient $S$ un sous-espace analytique d'un ouvert $\Omega$ de $c^{m}$, $r=\left(r_{1}, \ldots, r_{m}\right)$ tel que $D_{r} \subset \subset \Omega$, où $D_{r}$ est le polydisque ouvert de polyrayon $r$, et $s=\left(s_{1}, \ldots, s_{m}\right)$ où $0<s_{i}<r_{i}$. Alors les $\underline{O}\left(s \cap D_{r-t s}\right)$ forment une chaîne nucléaire d'algèbre. Si $U_{0}$ et $U$ sont des ouverts de stein de $c^{n}$ tels que $U \subset \subset U_{0}$ et $E$ un faisceau cohérent sur $S \times U_{0}$, cette chaśne nucléaire d'algèbre est transverse à $\underline{F}\left(\left(S \cap D_{r}\right) \times U\right)$.

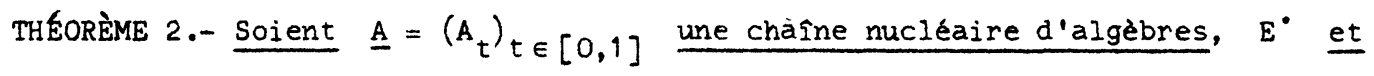
$F^{*}$ des complexes de Ao-modules de Fréchet nucléaires, bornés à droite, et $f^{*}: E^{*} \rightarrow F^{*}$ un morphisme de complexes. On suppose que $f^{*}$ induit des isomorphismes sur 1'homologie, que les $f^{n}$ sont $A_{0}$-sous-nucléaires et que $A$ est transverse aux $E^{n}$ et aux $F^{n}$. Alors, il existe un complexe $L^{\cdot}$ de $A_{1}$-modules tel que $L^{n}$ soit libre de type fini sur $A_{1}$, et un morphisme de complexes $h: L^{*} \rightarrow A_{1} \hat{\otimes}_{A_{0}} E^{\cdot}$ qui induit un isomorphisme sur I'homologie.

Lemme 3.- Soit $t_{0}<1$, soient $E^{*}$ et $F^{\cdot}$ deux complexes de $A_{t_{0}}$-modules de Fréchet nucléaires bornés à droite et acycliques en degré $>n$, et soit $f^{*}: E^{*} \rightarrow F^{*}$ un morphisme co complexes. On suppose que $f^{n}$ est $A_{t_{0}}{ }^{\text {-sous- }}$ nucleaire, que $f^{*}$ induit un isomorphisme $H^{k}\left(E^{*}\right) \rightarrow H^{k}\left(F^{*}\right)$ pour tout $k$, et que $A_{t}$ est transverse a $E^{k}$ et $F^{k}$ pour tout $k$ et tout $t \geq t_{0}$. Alors il existe $t_{1}<1$ tel que $H^{n}\left(E_{t_{1}^{*}}\right)$ et $H^{n}\left(F_{t_{1}}^{*}\right)$ soient des modules de type fini sur $A_{t_{1}}$, où $E_{t_{1}}^{*}=A_{t_{1}} \hat{\otimes}_{A_{t_{0}}} E^{\cdot}$ et $F_{t_{1}}^{*}$ de meme. 


\section{A. DOUADY}

$\mathrm{II}^{\mathrm{bis}-12}$

Démonstration. Les suites exactes $0 \rightarrow Z^{k}\left(E^{\cdot}\right) \rightarrow E^{k} \rightarrow Z^{k+1}\left(E^{\cdot}\right) \rightarrow 0$ montrent par récurrence descendante que $A_{t}$ est transverse à $Z^{k}\left(E^{\cdot}\right)$, et de meme à $z^{k}\left(F^{\circ}\right)$, pour $k \geq n$. L'application $f^{n}$ induit une application $A_{t_{0}}{ }^{-}$ sous-nucléaire $Z^{n}\left(E^{*}\right)$ dans $F^{n}$, dont $l^{\prime}$ image est contenue dans $Z^{n}\left(F^{*}\right)$. D'après la prop. 5 , pour $t_{0}<t^{\prime}<1$, l'application $z^{n}\left(E_{t^{\prime}}\right) \rightarrow z^{n}\left(F_{t^{\prime}}\right)$ induite par $f^{n}$ est $A_{t}$-sous-nucléaire. L'application $\left(d, f^{n}\right): F_{t^{\prime}}^{n-1} \oplus z^{n}\left(E_{t^{\prime}}^{\prime}\right) \rightarrow z^{n}\left(F_{t^{\prime}}^{*}\right)$ est surjective, et $\left(0, f^{n}\right)$ est $A_{t^{\prime}}-$ sous-nucléaire, donc pour $t^{\prime}<t_{1}<1$ l'application $d: F_{t_{1}}^{n-1} \rightarrow z^{n}\left(F_{t-1}^{\cdot}\right)$ a un conoyau de type fini sur $A_{t_{1}}$. C.Q.F.D.

Démonstration du Théorème. On va construire par récurrence descendante $t_{n}<1$, un A-module libre de type fini $L^{n}$ sur $A_{t_{n}}$, des morphismes $d^{n}: L^{n} \rightarrow L_{t_{n}}^{n+1}$ et $h^{n} \rightarrow E_{t_{n}}^{n}$ tels que :

1) $L_{(n)}: 0 \rightarrow L^{n} \rightarrow L_{t_{n}}^{n+1} \rightarrow \ldots$ soit un complexe et $h^{\cdot}$ un morphisme de ce complexe dans $E^{\cdot}$;

2) le "mapping cylinder" $M(n)$ de ce morphisme, défini par $M_{(n)}^{M^{k}}=E^{k} \oplus L^{k+1}$ pour $k \geq n-1$, soit acyclique en degré $\geq n$.

Supposons $L^{k}$, etc. construits pour $k \geq n+1$. Le "mapping cylinder" $\left.N_{(n+1}\right)$ de $f^{*} \circ n^{*}$ est également acyclique en degré $>n$, et le morphisme de $M(n+1)$ dans $N_{(n+1)}$ donné par $f^{*}$ est $A_{t}$-sous-nucléaire en chaque degré et induit un isomorphisme sur l'homologie. D'après le lemme 3 , il existe $t_{n}<1$ tel que $H^{n}\left(M_{t_{n}^{*}}\right)$ soit un $A_{t_{n}}$-module de type fini. On peut alors trouver un $A_{t_{n}}$-module libre de type fini $L^{n}$ et un morphisme $\left(\begin{array}{l}h^{n} \\ d^{n}\end{array}\right): L^{n} \rightarrow M^{n}=E_{t_{n}}^{n} \oplus L_{t_{n}}^{n+1}$ dont l'image est contenue dans $Z^{n}\left(M^{*}\right)$ et tel que l'image de $E^{n-1}+L^{n}$ soit $Z^{n}\left(M^{*}\right)$. Le module $L^{n}$, muni de $\left(h^{n}, d^{n}\right)$ répond
a la question.
C.Q.F.D. 
7. Démonstration du Théorème des images directes.

Soient $X$ et $S$ des espaces C-analytiques séparés, $\pi: X \rightarrow S$ un morphisme propre, $F$ un faisceau analytique cohérent sur $X$ et $s_{0}$ un point de $s$. On va montrer que les faisceaux $\underline{R}^{n} \pi_{*} \underline{F}$ sont cohérents au voisinage de $s_{0}$. On peut supposer que $S$ est un sous-espace analytique fermé d'un ouvert $\Omega$ de $c^{\mathfrak{m}}$ et $s_{0}=0$. On peut trouver un polydisque $D_{R} \subset \Omega$ et des familles $\left(x_{i}\right)_{i \in I}$, $\left(\varphi_{i}\right)_{i \in I}$ telles que $I$ soit un ensemble fini, que $\left(x_{i}\right)$ soit un recouvrement ouvert de $\pi^{-1}\left(D_{R} \cap S\right)$ et que $\varphi_{i}$ soit un isomorphisme au-dessus de $\Omega$ de $x_{i}$ sur un sous-espace analytique fermé de $D_{R} \times U_{i}$, où $U_{i}$ est un ouvert de stein de $C^{n_{i}}$. On peut trouver $D_{r_{0}} \subset \subset D_{R}$ et des ouverts de stein $w_{i} \subset \subset v_{i} \subset \subset U_{i}$ tels que, en posant $v_{i, r}=\varphi_{i}^{-1}\left(D_{r} \times v_{i}\right)$ et $w_{i, r}$ de meme, les $w_{i, r}$ forment un recouvrement de $\left.\varphi^{-1}\left(D_{r_{c}}\right\urcorner s\right)$. Soit $s=\left(s_{1}, \ldots, s_{m}\right)$ tel que $s_{i}<r_{n_{i}}$. posons $\left.r_{t}=r-t s, A_{t}=\right)\left(D_{r_{t}} \cap s\right), \underline{v}_{t}=\left(v_{i, r}\right)$ et $\underline{w}_{t}$ de meme. Alors les $A_{t}$ forment une chaine i ucléaire, et les complexes de cochaines alternées $C^{*}\left(\underline{v}_{0} ; F\right)$ et $C^{*}\left(W_{0} ; F\right)$ satis Oont aux hypothèses du Théoreme 2 en vertu du Théorème $B$, du Théorème de Lera et ce la Proposition .

Par suite, il existe un comp exe $\underline{-}$ de faisceaux libres de type fini sur $S \cap D_{r_{1}}$ et un morphisme $n: \underline{L}^{*}\left(S \cap D_{r_{1}}\right) \rightarrow C^{*}\left(\underline{V}_{1} ; \underline{F}\right)$ qui induit un isomorphisme de $H^{n}\left(S \cap D_{r_{1}}\right)$ sur $H^{n}\left(\varphi^{-1}\left(S \cap D_{r_{1}}\right) ; E\right)$ pour tout $n$, en notant $\underline{H}^{n}$ le faisceau d'homologie du complexe de faisceaux $\underline{L}^{*}$. Il résulte de la Prop. 2 et du Cor. de la Prop. 3 que, pour tout ouvert de Stein $S^{\prime}$ de $S$ contenu dans $s \cap D_{r_{1}}$, le morphisme $h$ donne un isomorphisme de $\underline{H}^{n}\left(S^{\prime}\right)$ sur $H^{n}\left(\varphi^{-1}\left(S^{\prime}\right) ; E\right)$. Par suite, $h$ définit un isomorphisme de $\underline{H}^{n}$, qui est cohérent, sur $\underline{R}^{n} \pi_{*} \underline{F}$, au-dessus de $S \cap D_{r_{1}}$, ce qui démontre le théorème. 
II ${ }^{\text {bis }}-14$

\section{A. DOUADY}

8. Le Théorème de semi-continuité. $\left(^{*}\right)$

Avec les hypothèses et notations du Théorème des images directes, pour $s \in S$, posons $\mathrm{X}(\mathrm{s})=\pi^{-1}(\mathrm{~s})$ et $\underline{F}(\mathrm{~s})=\underline{\underline{O}}_{\mathrm{X}}(\mathrm{s}) \otimes_{\underline{O}_{\mathrm{X}}} \underline{\mathrm{F}}$.

THÉORĖME 3.- $\underline{S i} \underline{E}$ est plat sur $S$, pour tout $k \in \mathbb{N}$, l'ensemble des $s \in S$ tels que $\operatorname{dim} H^{n}(X(s) ; \underline{F}(s)) \geq k$ est un sous-ensemble analytique de $S$.

Démonstration. Reprenons les notations du no 7. Il suffit de voir que, pour $s \in S \cap D_{r_{1}}$, le morphisme $n: \underline{L}\left(S \cap D_{r_{1}}\right) \rightarrow C^{*}\left(\underline{V}_{-1} ; \underline{F}\right)$, qui induit un isomorphisme sur I'homologie, définit un isomorphisme de $\mathrm{H}^{\mathrm{n}}(\underline{\mathrm{L}} \cdot(\mathrm{s}))$ sur $\mathrm{H}^{\mathrm{n}}(\mathrm{X}(\mathrm{s}) ; \underline{\mathrm{F}}(\mathrm{s}))$. or cela résulte du Cor. de la prop. 3 et du lemme suivant :

Lemme 4.- Avec les notations de la Prop. 2, si $\underline{F}$ est plat sur $S_{0}$, le module $\underline{F}(S \times U)$ est transverse $a \underline{0}_{S, S} \underline{m}_{S}$ pour tout $s \in S$.

La démonstration de ce lemme est analogue $\nRightarrow$ celle de la prop. 2 , en remarquan que $\underline{L} .(s)$ est une résolution de $\underline{F}(s)(U)$ car $\underline{F}$ est plat.

\section{BIBLIOGRAPHIE}

[1.] H. CARTAN et J.-P. SERRE - Un théorème de finitude concernant les variétés analytiques compactes, C. R. Acad. Sci. Paris, 237 (1953), p. 128-130.

[2] H. GRAUERT - Ein Theorem der Analytischen Garbentheorie und die modulräume Complexer Strukturen, Publ. Math. I.H.E.S., Bures-sur-Yvette, 1960.

[3] K. KNORR - Der Grauertsche Kohärenzsatz, Inventiones Math. 1970.

[4] KIEHL-VERDIER - Ein Einfacher Beweis des Koharensatzes von Grauert, Math. Ann. 195 (1971), p. 24-50.

[5] FORSTER-KNORR - Ein Beweis des Grauertschen Bildgarbensatzes nach idees von

B. Malgrange, Manuscripta Mathematica, Vol. 5, Fasc. 1, 1971.

[6] Par exemple : F. TREVES - Topological Vector spaces, Academic Press.

$\left(^{*}\right)$ La rédaction de ce numéro distribuée lors du séminaire contenait un énoncé faux du Th. 3. Je remercie P. Siegfried de me l'avoir signalé. 